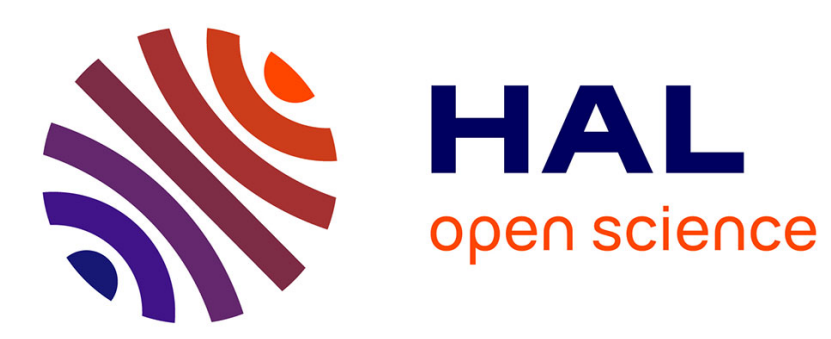

\title{
Nonlinear sculpturing of optical pulses with normally dispersive fiber-based devices
}

Christophe Finot, Ilya Gukov, Kamal Hammani, Sonia Boscolo

\section{To cite this version:}

Christophe Finot, Ilya Gukov, Kamal Hammani, Sonia Boscolo. Nonlinear sculpturing of optical pulses with normally dispersive fiber-based devices. Optical Fiber Technology, 2018, 45, pp.306. 10.1016/j.yofte.2018.08.007 . hal-01821853

\section{HAL Id: hal-01821853 \\ https://hal.science/hal-01821853}

Submitted on 22 Jun 2018

HAL is a multi-disciplinary open access archive for the deposit and dissemination of scientific research documents, whether they are published or not. The documents may come from teaching and research institutions in France or abroad, or from public or private research centers.
L'archive ouverte pluridisciplinaire HAL, est destinée au dépôt et à la diffusion de documents scientifiques de niveau recherche, publiés ou non, émanant des établissements d'enseignement et de recherche français ou étrangers, des laboratoires publics ou privés. 


\title{
Nonlinear sculpturing of optical pulses with normally dispersive fiber-based devices
}

\author{
Christophe Finot $^{1, *}$, Ilya Gukov ${ }^{2}$, Kamal Hammani ${ }^{1}$, and Sonia Boscolo ${ }^{3}$ \\ ${ }^{1}$ Laboratoire Interdisciplinaire Carnot de Bourgogne, UMR 6303 CNRS-Université de \\ Bourgogne-Franche-Comté, 9 avenue Alain Savary, BP 47870, 21078 Dijon Cedex, France \\ ${ }^{2}$ Moscow Institute of Physics and Technology \& Skolkovo Institute of Science and Technology, \\ Moscow, Russia \\ ${ }^{3}$ Aston Institute of Photonic Technologies, School of Engineering and Applied Science, Aston \\ University, Birmingham B4 7ET, United Kingdom \\ * Corresponding author: \\ E-mail address: christophe.finot@u-bourgogne.fr \\ Tel.: +33 380395926
}

\begin{abstract}
We present a general method to determine the parameters of nonlinear pulse shaping systems based on pulse propagation in a normally dispersive fiber that are required to achieve the generation of pulses with various specified temporal properties. The nonlinear shaping process is reduced to a numerical optimization problem over a three-dimensional space, where the intersections of different surfaces provide the means to quickly identify the sets of parameters of interest. We also show that the implementation of a machine-learning strategy can efficiently address the multi-parameter optimization problem being studied.
\end{abstract}

Keywords: Nonlinear shaping, machine learning, nonlinear fiber optics 


\section{Introduction}

In recent years, there has been a growing interest from the photonics community in the generation of non-conventional optical waveforms at repetition rates of several $\mathrm{GHz}$ because of their applications in all-optical signal processing and microwave signal manipulation. While sinusoidal, Gaussian and hyperbolic secant intensity profiles are now routinely produced by modulators or mode-locked lasers, other signal waveforms such as parabolic, triangular or flat-top pulse shapes remain rather hard to synthesize. Advances in fiber lasers have indicated promising ways to produce such waveforms [1-3], but the tunability of these lasers in terms of pulse repetition rate remains quite low and their experimental implementation and stability may require further work. Different approaches to the generation of specialized waveforms have also been explored. A first class of methods is based on photonic generation using special Mach-Zehnder modulator architectures [4, 5], microwave photonic filters [6] or frequency-to-time conversion [7, 8]. These methods can produce relatively (a few tens of picoseconds) long pulses. Another class of approaches aims at the synthesis of the target waveform directly in the frequency domain by adjustment of the amplitude and phase of different coherent spectral lines [9-11]. However, if the spectral lines involved are limited in number, the achievable duty cycle of the resulting pulse train will also be restricted. This limitation can be overcome by the use of ultra-short input pulses from a mode-locked laser. Pulse shaping using the Fourier-domain approach can indeed transform an ultra-short pulse into the desired shape, the transfer function in the frequency domain being the ratio of the target field distribution to the input field. In this context, picosecond and femtosecond pulse shaping has been achieved by use of spatial light modulators [12,13], super-structured fiber Bragg gratings [14], acousto-optics devices [15], and arrayed waveguide gratings [16]. Though being powerful and flexible, as the numerous successes of the fore-mentioned methods have demonstrated, the linear pulse shaping strategy has the intrinsic drawback that the bandwidth of the output spectrum is determined by the bandwidth of the input spectrum. Indeed, a linear manipulation cannot increase the pulse bandwidth, and so to create shorter pulses nonlinear effects must be used. In addition, a linear pulse shaper can only subtract power from the frequency components of the signal while manipulating its intensity, thereby potentially making the whole process power inefficient. The combination of third-order nonlinear processes and chromatic dispersion in optical fibers can provide efficient new solutions to overcome the drawbacks of linear pulse shapers [17]. In particular, it has been demonstrated that it is possible to take advantage of 
the progressive nonlinear reshaping of conventional laser pulses that occurs upon propagation in a normally dispersive fiber to generate various advanced temporal waveforms, including parabolic [18-21] and triangular [22-26] profiles.

However, the determination of the optimal parameters of a nonlinear fiber system to achieve desired pulse characteristics is more complex than that involved in linear spectral shaping, where only the input and target waveforms are required. Indeed, the nonlinear shaping depends on both the input pulse condition and the fiber properties. In previous works, we have proposed and validated some rules for the design of nonlinear pulse shaping fiber schemes [18, 22, 26], but without imposing any requirement on the output pulse characteristic parameters such as the pulse duration, for example. To the best of our knowledge, no general method for the design of fiberbased nonlinear pulse shaping has been developed to date, enabling the identification of the optimal working parameters for the generation of pulses with various prescribed characteristics. In this paper, we present such approach, which provides a comprehensive exploration of the possibilities offered by fiber-based nonlinear pulse shaping and the determination of the the operational conditions within the space of system parameters for the formation of pulses with different, simultaneously optimized temporal features. After describing the degrees of freedom available in the system and the numerical procedure used for the characterization and optimization of the nonlinear shaping process, we illustrate our proposed approach through the examples of the generation of parabolic, triangular and rectangular waveforms with different pulse durations and time-bandwidth products. We also show that the multi-parameter optimization problem being considered can be efficiently addressed by using the machine-learning method of neural networks (NNs).

\section{Principle and situation under investigation}

In this section, we set up the problem to solve and outline the numerical procedure that we implement to deal with this problem.

\subsection{Principle of nonlinear pulse shaping and available degrees of freedom}

Nonlinear shaping in a normally dispersive fiber involves many degrees of freedom. A scheme for nonlinear shaping typically comprises two stages: a pre-chirping stage followed by a nonlinear 
propagation stage. Within such scheme, an initial pulse $\psi_{0}(t)$ with a peak power $P_{0}$ and a full-width at half maximum (fwhm) duration $T_{i n}$ is first propagated through a dispersive medium, such as a pair of diffraction gratings, a prism pair [27], a segment of hollow core or standard fiber with very low nonlinearity $[23,24]$. This linear propagation imprints a parabolic spectral phase onto the pulse, which is characterized by a chirp coefficient $C_{0}$ that can be positive or negative depending on the group-velocity dispersion (GVD) of the medium being normal or anomalous. The so obtained chirped pulse is then propagated through a normally dispersive fiber that reshapes both its temporal and spectral intensity profiles. According to the initial conditions of the input pulse, the initial stage of nonlinear dynamics in the fiber, where Kerr-induced self-phase modulation (SPM) dominates over GVD, may be very different. Indeed, input pulses with a negative chirp coefficient will experience spectral compression as a result of SPM [15, 28, 29], whereas for initially positively chirped (or Fourier transform-limited) pulses, spectral broadening will drive the nonlinear dynamics and eventually lead to optical wave-breaking [30]. Moreover, propagation in the nonlinear fiber is impacted by both GVD and SPM effects, which are characterized by the respective coefficients $\beta_{2}$ and $\gamma$. The length of the fiber $L$ is also a crucial parameter that must be carefully selected. Therefore, from an experimental standpoint and for a given initial pulse waveform, at least six parameters can be adjusted to obtain the combination that is fit for purpose.

Note that in this paper we consider the simplest model of fiber propagation only including the dominant physical effects of the system. Indeed, higher-order linear effects such as third- or fourth-order dispersion, and nonlinear effects such as self-steepening or intra-pulse Raman scattering have negligible impact on pulses with picosecond-range durations as the ones being considered here. Note also that our discussion does not embrace the additional pulse shaping possibilities offered by advanced fiber designs such as fibers with distributed gain or longitudinally varying parameters [31-33]. Furthermore, we would like to emphasize that the focus of the present study is on pulse shaping in fibers with normal GVD. Anomalously dispersive fibers may sustain very different pulse dynamics, characterized by the emergence of solitonic structures that can be trickier to handle [34]. To summarize, even in the simplest configuration being studied, there are six physical parameters that must be used as input data for the nonlinear shaping problem, namely, $\left(C_{0}, T_{0}, P_{0}, \beta_{2}, \gamma, L\right)$, where $T_{0}$ is a characteristic temporal value of the input pulse. 


\subsection{Features of the target pulses}

The independent variation of the six system's parameters discussed above provides access to a large variety of output pulse temporal features. In addition to markedly different pulse shapes (i.e., parabolic, triangular and rectangular waveforms) whose generation has been studied in previous works [18, 22, 27], one can also achieve a very broad range of output pulse durations, bearing different levels of chirp. It is worth noting that contrary to what typically occurs upon nonlinear propagation in a fiber with anomalous GVD, using a normally dispersive fiber as the nonlinear shaping element favors the formation of pulses that are longer than the input pulses.

Different approaches are possible to characterize the pulse shape [18, 22, 35]. Here we compute the parameter of misfit $M$ between the pulse temporal intensity profile $I_{N}$ and the target shape fit $I_{T}$ :

$$
M^{2}=\int\left(I_{N}-I_{T}\right)^{2} d t / \int I_{N}^{2} d t
$$

We also consider the coefficient of excess kurtosis as a measure of shape [35]. We use the fwhm pulse duration $T_{\text {out }}$ as a measure of the temporal extent of the pulse. The level of chirp present in the pulse is quantified by computing the Strehl ratio $S$, defined as the ratio of the maximum spectral brilliance of the actual pulse to the spectral brilliance obtained assuming a flat temporal phase of the pulse. Therefore, $S$ is comprised between 0 and 1, with 1 defining a Fourier transform-limited waveform. The bandwidth at fwhm $F_{\text {out }}$ of the frequency spectrum of the pulse is also used as a descriptor of the chirp.

\subsection{Pulse propagation model}

Pulse propagation in the fiber system follows the standard nonlinear Schrödinger equation (NLSE) \{Agrawal, $2006 \# 8\}$ :

$$
i \frac{\partial \psi}{\partial z}-\frac{\beta_{2}}{2} \frac{\partial^{2} \psi}{\partial t^{2}}+\gamma|\psi|^{2} \psi=0
$$

where $\psi(z, t)$ is the complex envelope of the pulse, $z$ is the propagation coordinate and $t$ is the retarded time. Note that the effects of linear loss can be neglected given the very low loss of silica fibers in the telecommunication wavelength window. As mentioned earlier, here we also neglect 
higher-order linear and nonlinear effects as the leading-order behavior is well approximated by Eq. (2).

The linear dispersive element can be described by Eq. (2) with $\gamma=0$. As a result of GVD, the initial pulse acquires a parabolic phase in the spectral domain:

$$
\psi=\psi_{0} \exp \left(i \frac{C_{0} \omega^{2}}{2}\right)
$$

where $\psi$ denotes the Fourier transform of the pulse envelope, and the chirp coefficient $C_{0}$ equals the cumulative GVD. This spectral phase leads to temporal broadening of the pulse and the development of a chirp in the time domain, which is linear when $C_{0}$ is high (i.e., over far-field evolution). This stretched pulse then evolves in the nonlinear fiber according to Eq. (2).

It is useful to normalize Eq. (2) by introducing the dimensionless variables: $u=\psi / \sqrt{P_{0}}, \xi$ $=z / L_{D}, \tau=t / T_{0}$, and write it in the form

$$
i \frac{\partial u}{\partial \xi}-\frac{1}{2} \frac{\partial^{2} u}{\partial \tau^{2}}+N^{2}|u|^{2} u=0
$$

where $L_{D}=T_{0}{ }^{2} /\left|\beta_{2}\right|$ and $L_{N L}=1 /\left(\gamma P_{0}\right)$ are the respective dispersion length and nonlinear length associated with the pulse at the input to the system, and the parameter $N$ ('soliton-order' number) is introduced as $N^{2}=L_{D} / L_{N L}$. This way, the search for the best system parameters to achieve specified output pulse characteristics is trimmed to a three-dimensional optimization problem in the space of $(C, N, \xi)$, where $C=C_{0} T_{0}$ is the normalized chirp coefficient of the pulse at the entrance of the fiber. Hence, the parameter search is apparently made significantly more efficient, and for a specific selected set $(C, N, \xi)$ there are many groups of practical parameters suitable the defining equations of $C, N$, and $\xi$.

\subsection{Numerical procedure}

Compact analytical or semi-analytic models are valuable for an improved intuitive understanding

of the nonlinear pulse evolution in optical fibers [36, 37]. However, being approximate, such methods typically do not allow for precise design optimization over vast parameter ranges. Shaping methods based on calculating the reverse propagation of the desired pulse shape in the fiber are also rather restricted [38]. Therefore, we chose to base our discussion on numerical 
solution of the pulse-propagation problem based on the NLSE. We use a standard symmetric splitstep Fourier algorithm [34] for this purpose. We use both pulses with a Gaussian intensity profile $\psi_{0}(t)=\sqrt{P_{0}} \exp \left(-t^{2} / 2 T_{0}^{2}\right)$ and a hyperbolic secant profile $\psi_{0}(t)=\sqrt{P_{0}} \operatorname{sech}\left(t / T_{0}\right)$ as initial conditions for our study. For the Gaussian pulse we explore the parameter space defined by $C$ between -4.8 and 4.8, $\xi$ between 0 and 27.6 and $N$ between 0 and 7.6. For the hyperbolic secant pulse the search range is $C$ between -6.5 and $6.5, \xi$ between 0 and 31 and $N$ between 0 and 7 .

In a first approach we perform high-resolution (HR) numerical simulations of the propagation model using parallel computing on the CPU of a standard personal computer. We use a grid of 200 $\times 200 \times 200$ points to discretize the parameter space, and for each point we record the output pulse characteristics described in the previous section. However, such studies based on extensive numerical simulations are in general a costly exercise. The general problem of optimization towards a target operational regime in a complex multi-parameter space can be intelligently addressed by implementing machine learning strategies. Genetic algorithms have recently been used to solve problems of nonlinear optical shaping in passive fibers [39-41]. Here we explore the possibilities offered by artificial neural networks, which have already been applied in the context of ultrafast fiber lasers [42]. Therefore, in a second approach, we produce a reduced-size set of 50 $\times 50 \times 50$ data points from full simulations of the governing equation and then train a regression model based on a neural network algorithm on this data to identify the target output pulse states across the full range of parameter variation. We use a feed-forward neural network relying on the Levenberg-Marquardt back propagation algorithm and involving a hidden layer of 125 to 200 neurons. This neural network is a part of the Matlab neural network toolbox.

\section{Generation of nearly transform-limited parabolic pulses of prescribed duration}

In order to illustrate our general approach to the design of fiber-based nonlinear pulse shaping, we first discuss the generation of parabolic waveforms. The use of nonlinear propagation in fiber to create parabolic-shaped pulses has been extensively studied in the literature, with an emphasis on fiber amplifiers [43, 44]. An arbitrary pulse inserted in an active (gain) or dispersion-decreasing fiber (where a longitudinal decrease of the normal dispersion emulates linear gain) is nonlinearly attracted toward an asymptotically evolving parabolic pulse subject to certain relations (scaling) between pulse amplitude, duration and chirp parameter. While the pulse duration of this self- 
similar parabolic solution can be controlled to some extent, the formed pulse is highly chirped, thereby featuring a time-bandwidth product that is significantly larger than the Fourier-transform limit value. Conversely, here we target a nearly transform-limited output pulse from the passive fiber, hence a Strehl ratio close to 1. Starting with an input Gaussian pulse, we consider that the desired parabolic pulse form has been achieved when the misfit parameter becomes equal to or lower than a sufficiently small value, which is set to 0.045 as an example. We also target a specific temporal duration of the output pulse, i.e. a duration ten times larger than the input one: $T_{\text {out }}=10$ $T_{i n}$.

\subsection{Determination of the optimal parameters}

We use a graphical method to find the combinations of values for the parameters $C, N$, and $\xi$ that support the formation of pulses with the required characteristics. We first determine the region of the three-dimensional parameter space that leads to a parabolic profile. The results are plotted on Fig. 1(a) and the obtained complex surface indicates that various combinations of parameter values are fit for purpose. In Fig. 1(b), we have isolated the region of the parameter space that leads to the desired output pulse duration. The intersection of these two surfaces provides the region of space that fulfills the requirements on the pulse shape and duration simultaneously, as shown in Fig. 2(a). Note that, instead of using two separate surfaces, we could have directly computed the misfit parameter to a parabolic shape with the desired duration. However, this approach would be far less flexible as a change in the target output pulse would require a recalculation of the whole parameter space. Separating the various output parameters is therefore much more efficient as once the three-dimensional space has been calculated from a given input pulse waveform, it can be conveniently used to work a wide range of target output pulse features out. 

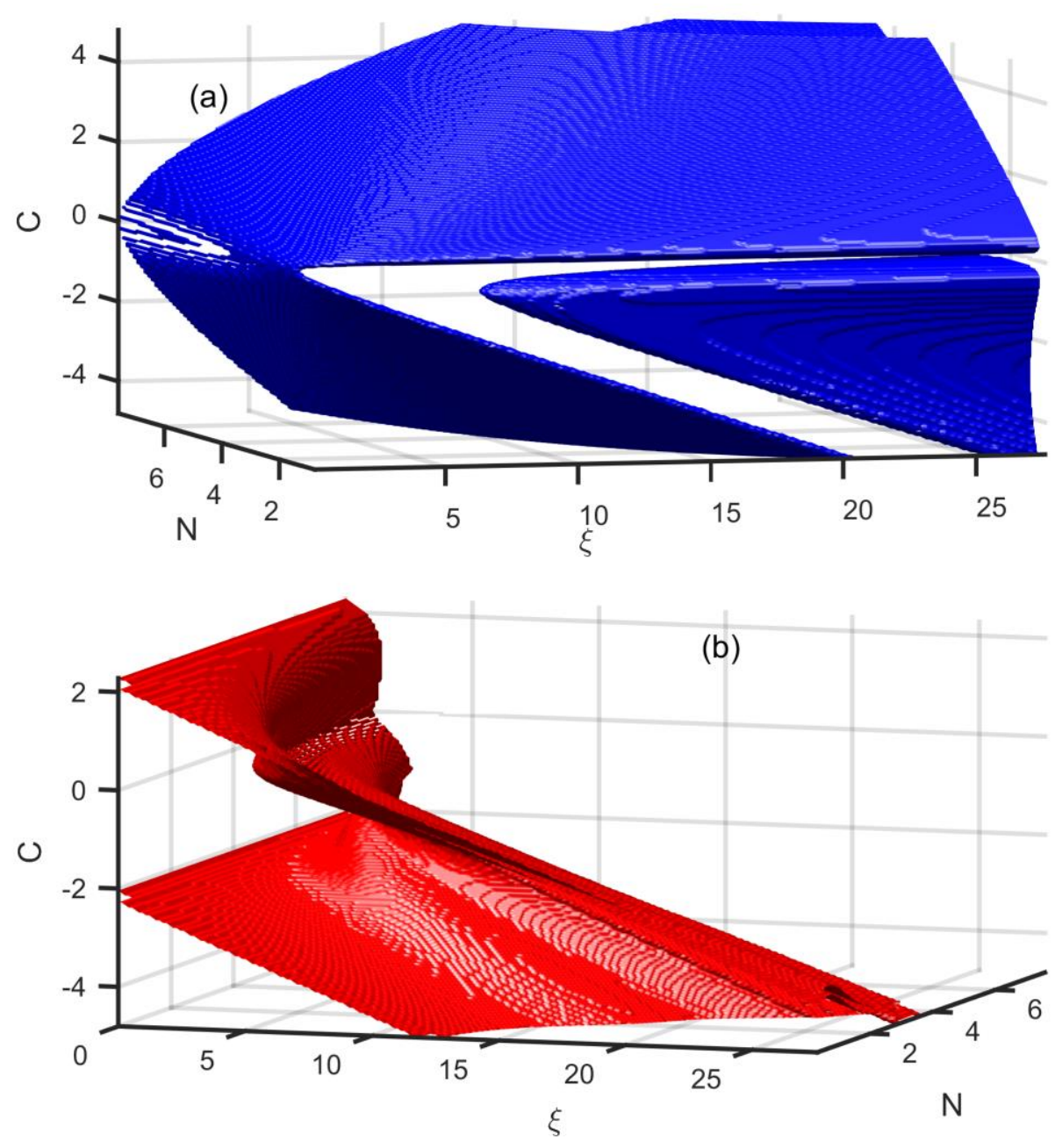

Figure 1: Surfaces in the three-dimensional parameter space that enable: (a) the formation of a pulse with a misfit parameter $M$ to a parabolic shape less than or equal to 0.045 , (b) an output temporal duration of $T_{\text {out }}=10 T_{\text {in }}$.

We can see in Fig. 2 that there are three different regions of parameter space that support the formation of a parabolic pulse with the desired duration. The region featuring normal input chirping corresponds to a transient state of the nonlinear dynamic pulse evolution in the fiber toward wave breaking $[18,30,45]$. The region featuring low input powers ( $N$ below 2$)$ and large propagated lengths corresponds to a long-term far-field evolution regime in the fiber characterized by the formation of pulses of a spectronic nature [20, 21, 46]. Finally, the region featuring anomalous input chirping and rather high input powers underpins nonlinear pulse dynamics dominated by a spectral compression process [29]. Figure 2 also contains information on the level of chirp present in the formed pulses. Indeed, the colormap used to display the crossings of 
parameter surfaces indicates the values of the Strehl ratio of the pulses. Therefore, the optimum parameter region is apparently the one that involves spectral compression dynamics.
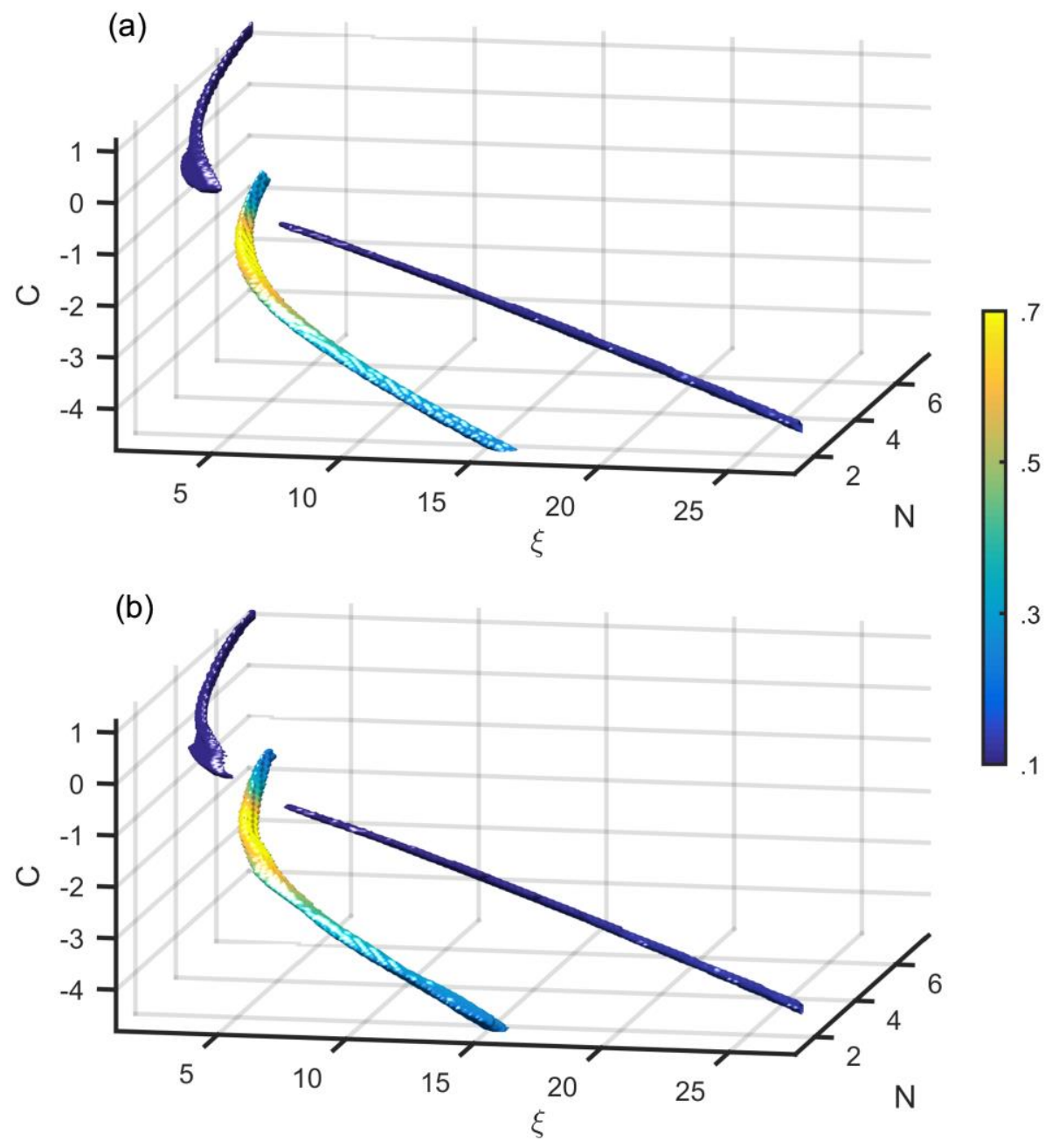

Figure 2: Crossings of parameter surfaces supporting the formation of a parabolic waveform with duration of $10 \mathrm{ps.}$ The results obtained from HR numerical simulations of the NLSE propagation model [subplot (a)] are compared with the results generated by the neural network regression model [subplot (b)]. The colormap indicates the Strehl ratio's values of the resulting pulses.

Figure 3 shows the temporal and spectral characteristics of the pulse generated at the point $(C=$ $-2, N=5, \xi=3$ ). We can see that the pulse has the desired waveform and temporal duration. Furthermore, the spectral intensity profile of the pulse is very close to that characteristic of a transform-limited parabolic pulse, and is significantly narrower than the input pulse spectrum. 

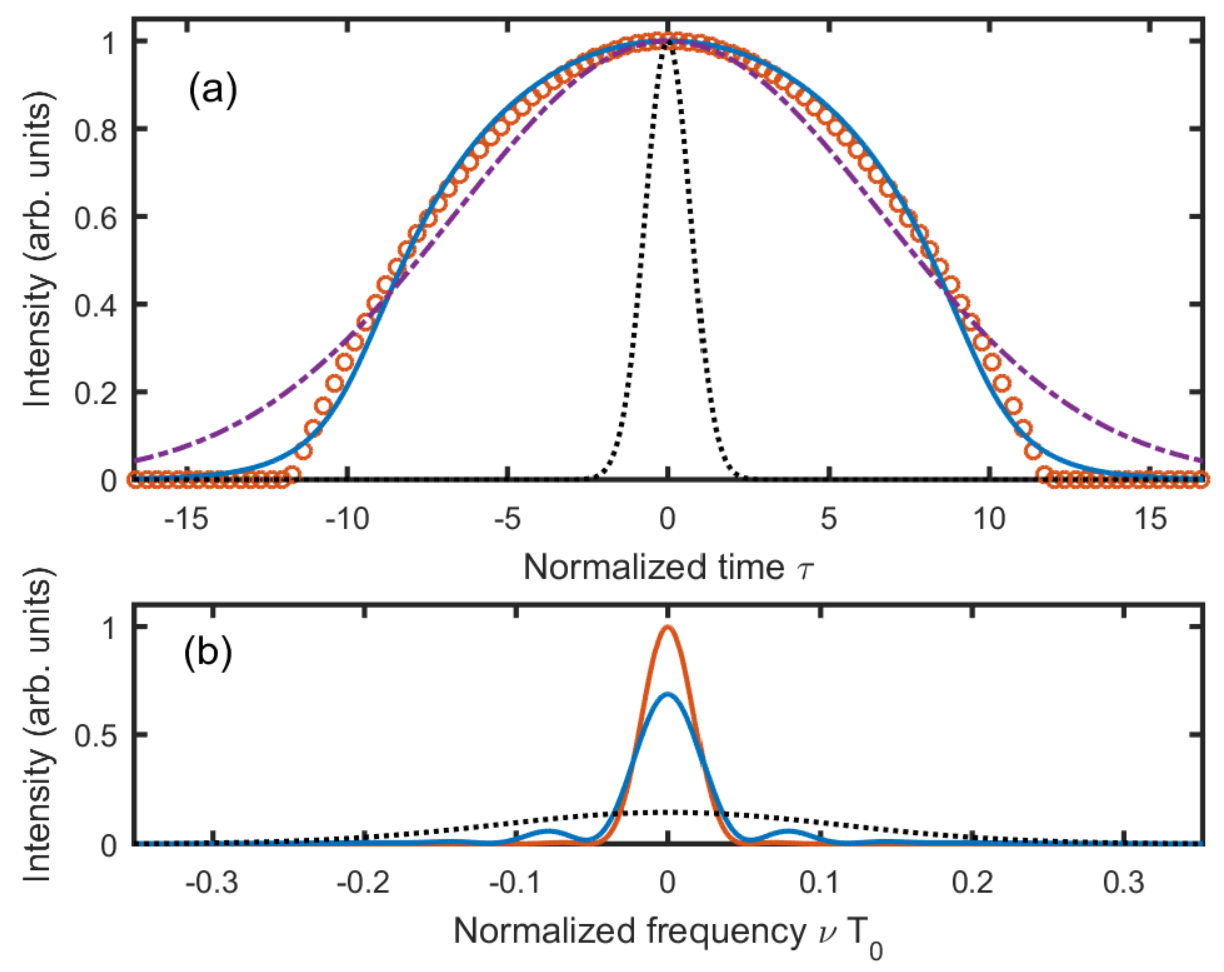

Figure 3: (a) Temporal and (b) spectral intensity profiles of the pulse generated at the point $(C=-2, N=5, \xi=3)$ (blue curves). Also shown are the target temporal profile (red open circles), the pulse spectrum obtained assuming a flat temporal phase (solid red curve), the temporal and spectral profiles of the input pulse to the system (dotted black curves), and the temporal profile obtained after the initial dispersive propagation stage (dash-dotted purple curve).

\subsection{Results of neural network regression}

The parameter regions corresponding to the formation of parabolic pulses of the desired width produced with the neural network training method are plotted on Fig. 2(b), and show good agreement with the results of HR numerical simulations of the NLSE propagation model. To ascertain how accurate are the predictions from the regression model, we have plotted on Fig. 4(a) a closed-up view of a map representing a two-dimensional slice of the three-dimensional space evaluating the misfit parameter to a parabolic shape as a function of $N$ and $\xi$ for $C=-2$. We see that initially the training data of the NN [panel (a2)] are poorly defined compared to the HR data [panel (a1)]. However, no difference can be seen with the naked eye between the map generated by the neural network [panel (a3)] and the map produced by HR numerical simulations. The mean absolute error is well below 0.01 [Fig. 4(b)]. 


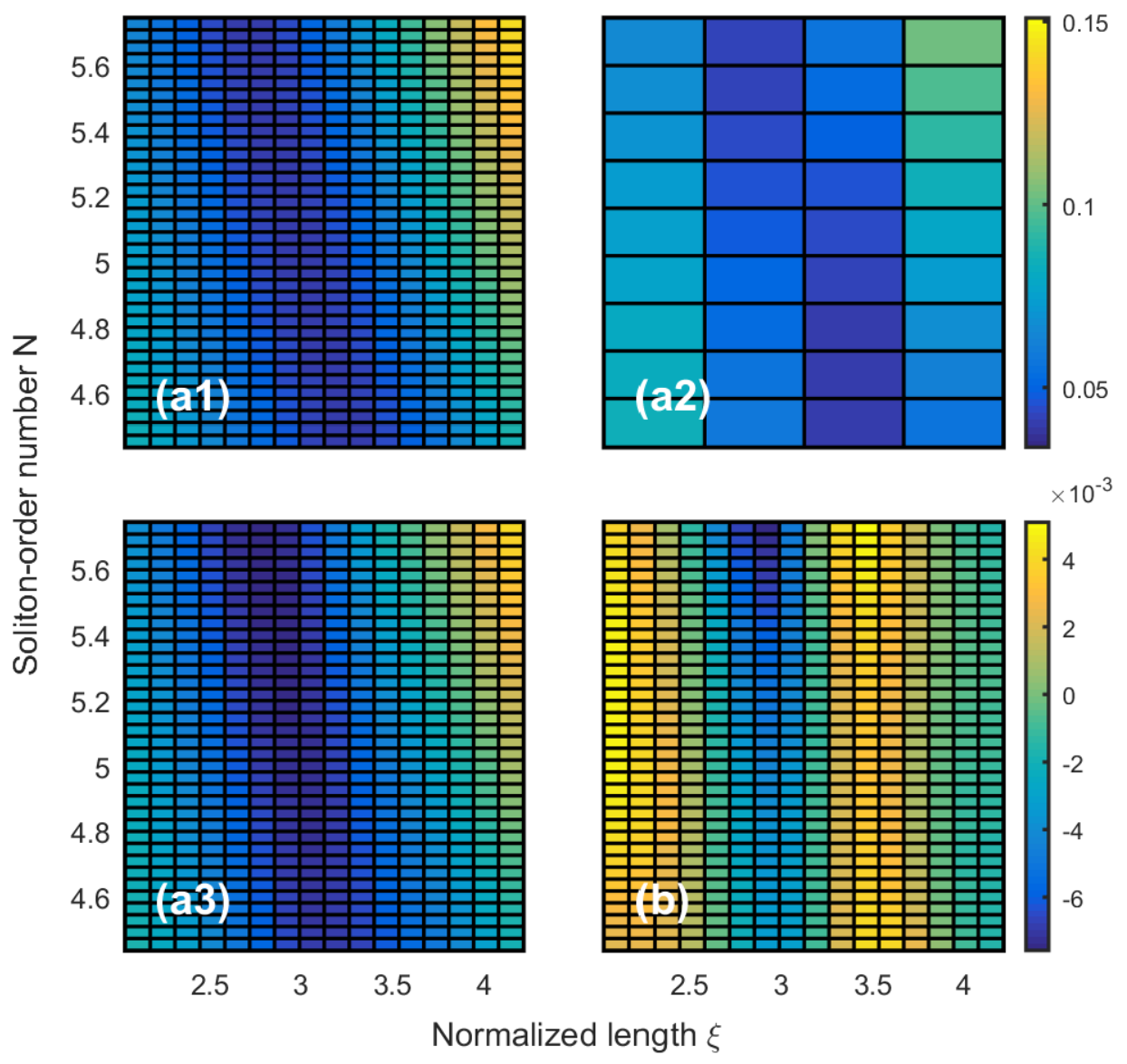

Figure 4: (a) Closed-up view of the two-dimensional map of misfit parameter values in the vicinity of $N=5$ and $\xi=$ 3 for $C=-2$. The map of expected values $M_{E, i}$ as produced by HR numerical simulations of the NLSE is shown in panel 1. Panels 2 and 3 show the training data and the map of values $M_{P, i}$ generated by the neural network, respectively. (b) Map of error values $M_{P, i}-M_{E, i}$.

The results in Fig. 4 assess the ability of the regression model to interpolate from the training data set to previously unseen data. It is also interesting to evaluate the ability of the model to continue to make good predictions, or at least not to fail catastrophically, when the training data covers only a limited range of the data domain. For this extrapolation task, we focus on the temporal duration and Strehl ratio of the output pulse from the fiber for a fixed input chirp parameter $C$. The training of the network is restricted to the data obtained for the same parameter ranges as those used in the interpolation task, i.e. $C$ from -4.8 to 4.8 , soliton-order number $N$ from 0 to 7.6 , and normalized propagated length $\xi$ from 0 to 27.6, but now we ask the network to make predictions for $C=-6$, $N$ values up to 10 , and $\xi$ values up to 36 . Hence, the new $N$ and $\xi$ ranges are $33 \%$ larger than the 
ranges used to produce the training data. The predictions from the regression model show qualitative agreement with the results of HR simulations of the NLSE carried out over the extended parameter domain (Fig. 5). However, deviations from the simulation results are this time noticeable, and rare but unphysical predictions, such as negative temporal duration or Strehl ratio values, can also occur. Notwithstanding, the parameter regions supporting high Strehl ratios and long or short pulse durations are rather well predicted by the neural network. This indicates that one could advantageously use the extrapolation generalization performed by the network as a first guess for more refined numerical simulations over the significant parameter subsets.

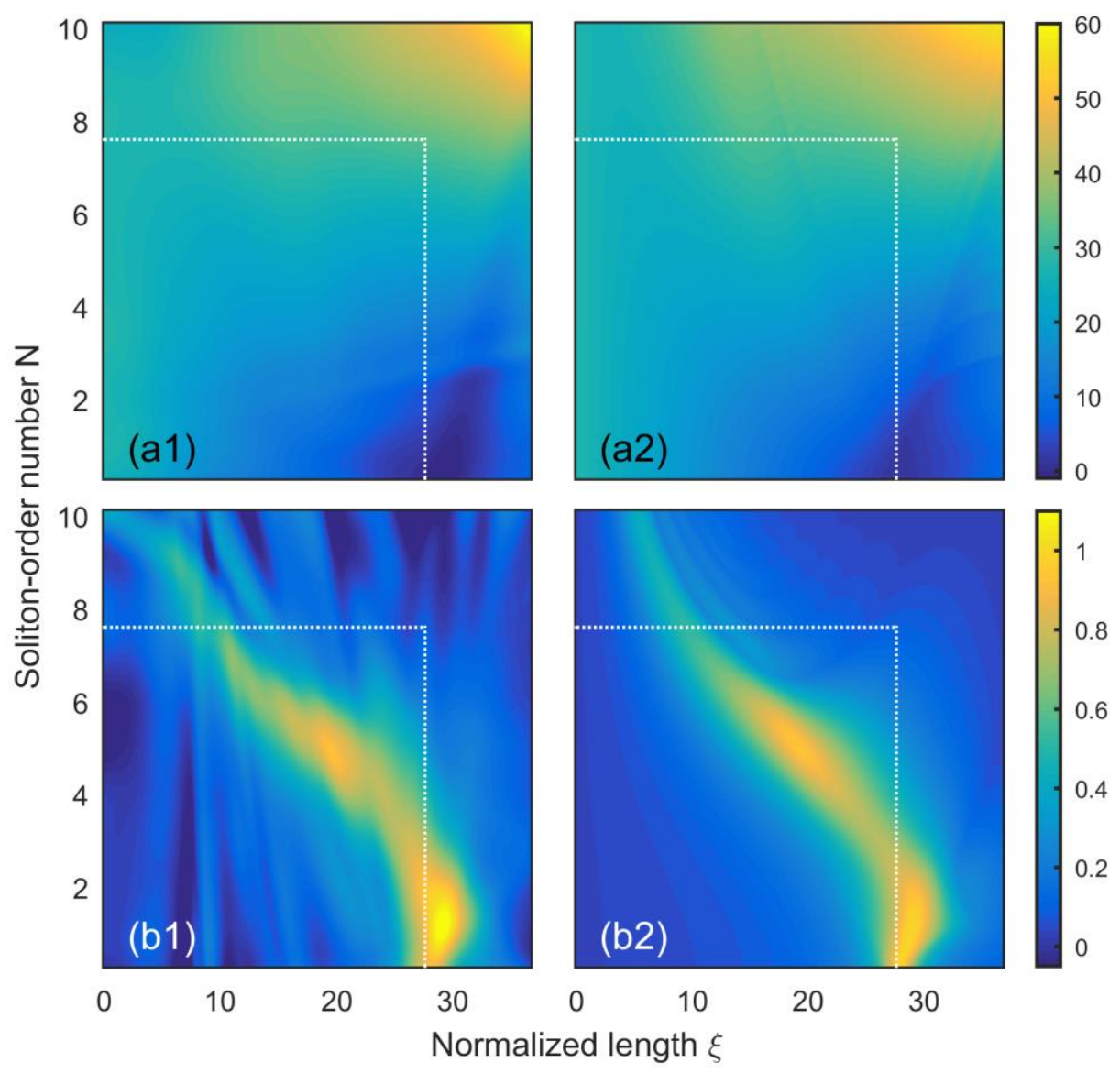

Figure 5: Two-dimensional maps of (a) temporal duration values and (b) Strehl ratio values for $\mathrm{C}=-6$ illustrating the extrapolation performance of the neural network. The maps generated by the neural network (panels 2) are compared with the maps produced by HR numerical simulations of the NLSE (panels 1). The dotted lines delimit the parameter region used to obtain the training data (along with the range of $C$ values from -4.8 to 4.8 . 


\section{Examples of generation of other pulse shapes}

\subsection{Triangular waveform generation}

As a second example of nonlinear pulse shaping in the fiber, we discuss the generation of triangular waveforms with a prescribed duration, which we choose to be, for instance, fifty times larger than the input duration. The surface in the three-dimensional parameter space corresponding to a low misfit parameter to a triangular shape ( $M$ below 0.015$)$ is plotted in blue color on Fig. 6(a). We can see that, contrary to the formation of parabolic pulses in the fiber, the parameter region that supports reshaping of the initial Gaussian pulse into a triangular waveform is quite narrow and, as discussed in previous works [22-24, 26], is characterized by anomalous input chirping. The surface corresponding to $T_{\text {out }}=50 T_{\text {in }}$ is plotted is red color and intersects the blue surface. The intersection region plotted on Fig. 6(b) is rather narrow, thereby enabling a significantly more limited choice of parameter combinations as compared to the parabolic case. The input power should be relatively high to observe reshaping to a triangular pulse ( $N$ higher than 6.5). We can also see in Fig. 6(b) that for a given pulse duration, the spectral width of the output pulse can vary significantly depending on the initial pulse chirp and, if we target minimal extent of the pulse spectrum, we should favor a high input chirp. 

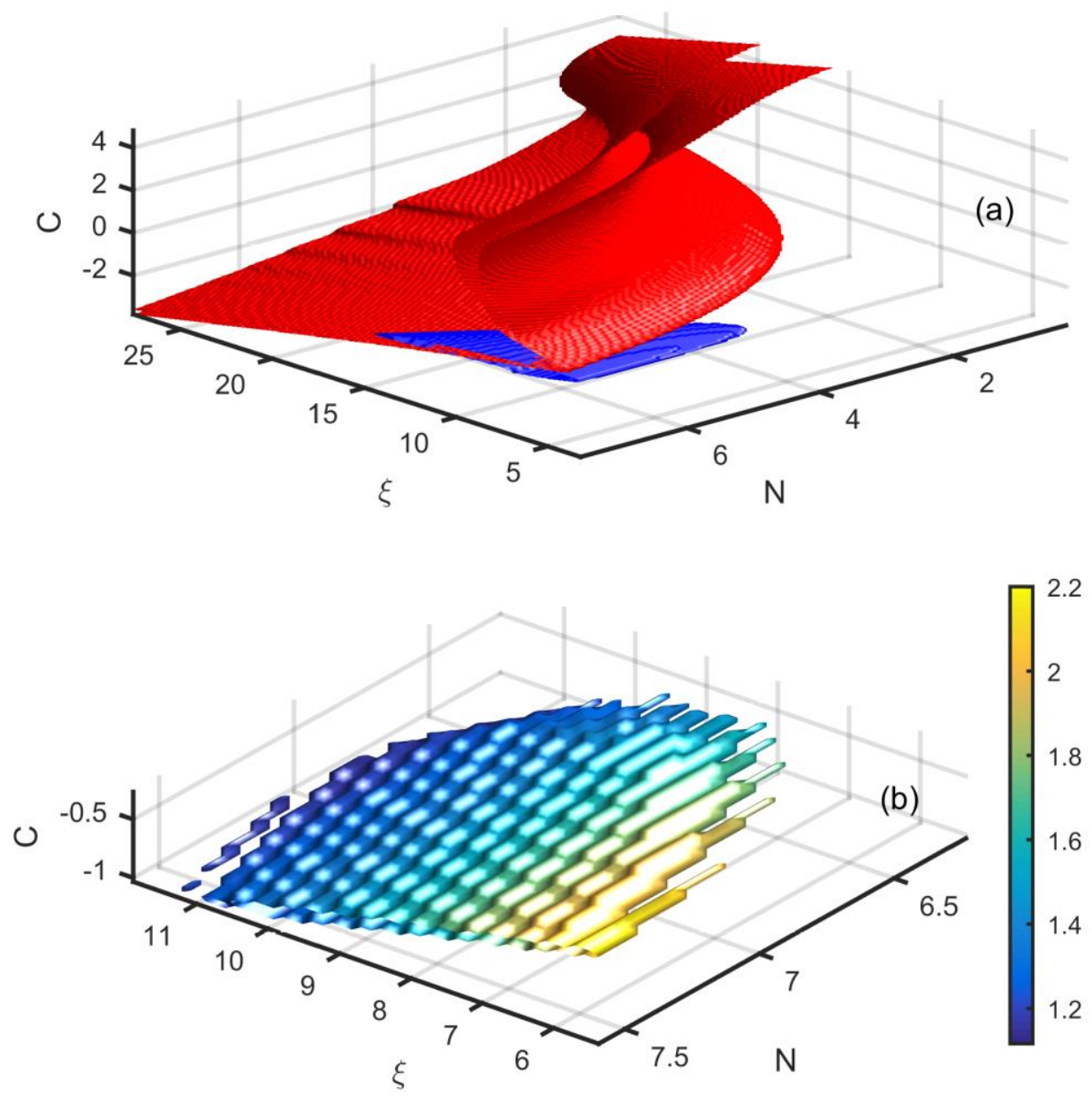

Figure 6: (a) Surfaces in the three-dimensional parameter space that enable the formation of a triangular waveform (blue) and an output temporal duration of $T_{\text {out }}=50 T_{\text {in }}$ (red). (b) Crossing of the two surfaces. The colormap indicates the spectral width's values of the resulting pulses.

Figure 7 shows that using the parameter set $C=-1, N=7.25$ and $\xi=11.5$ leads to clear reshaping of the initial pulse into a pulse with the target triangular shape and duration. However, as shown in the panel (b), the formed pulse is far from being transform-limited [panel (b)]; on the contrary, its spectrum has widened significantly over the initial spectrum. 


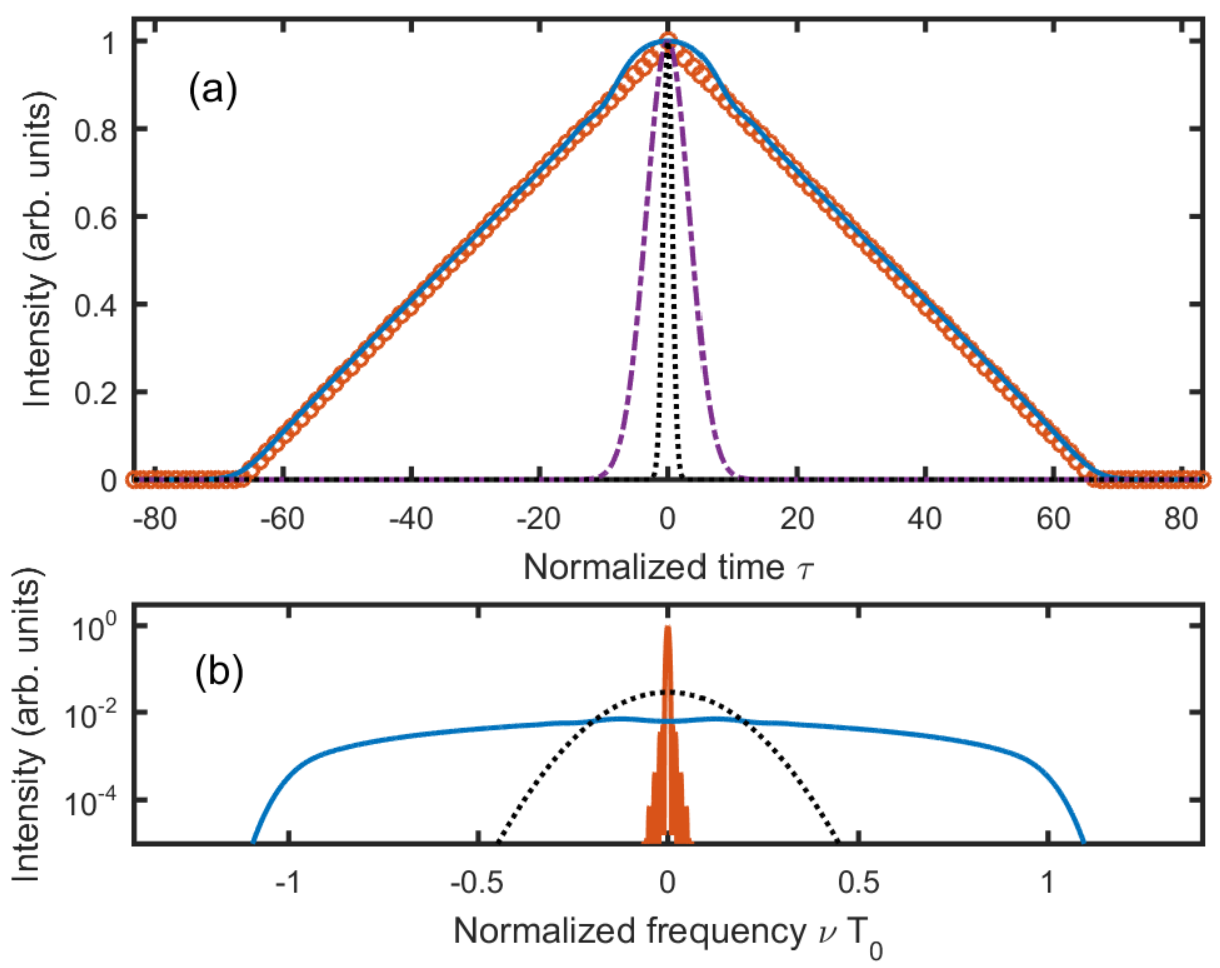

Figure 7: (a) Temporal and (b) spectral intensity profiles of the pulse generated at the point $(C=-1, N=7.25, \xi=$ 11.5) (blue curves). Also shown are the target temporal profile (red open circles), the pulse spectrum obtained assuming a flat temporal phase (solid red curve), the temporal and spectral profiles of the input pulse to the system (dotted black curves), and the temporal profile obtained after the initial dispersive propagation stage (dash-dotted purple curve).

\subsection{Rectangular waveform generation}

In this section we discuss the possibility of achieving nearly-rectangular waveforms at the output of the fiber. We start here from a hyperbolic secant pulse and we target a rectangular output profile with a temporal duration twenty times larger than the input one. Instead of the misfit factor, in this example we use the excess kurtosis as a measure of shape, hence we search pulses with an excess kurtosis around -1 . The non-empty intersection of the corresponding surfaces in the threedimensional parameter space (Fig. 8) indicates that the requirement on the output pulse duration is compatible with the requirement on the excess kurtosis coefficient. With respect to the level of chirp present in the output pulse, we can see that high Strehl ratio is attainable with an initial anomalous chirp. 

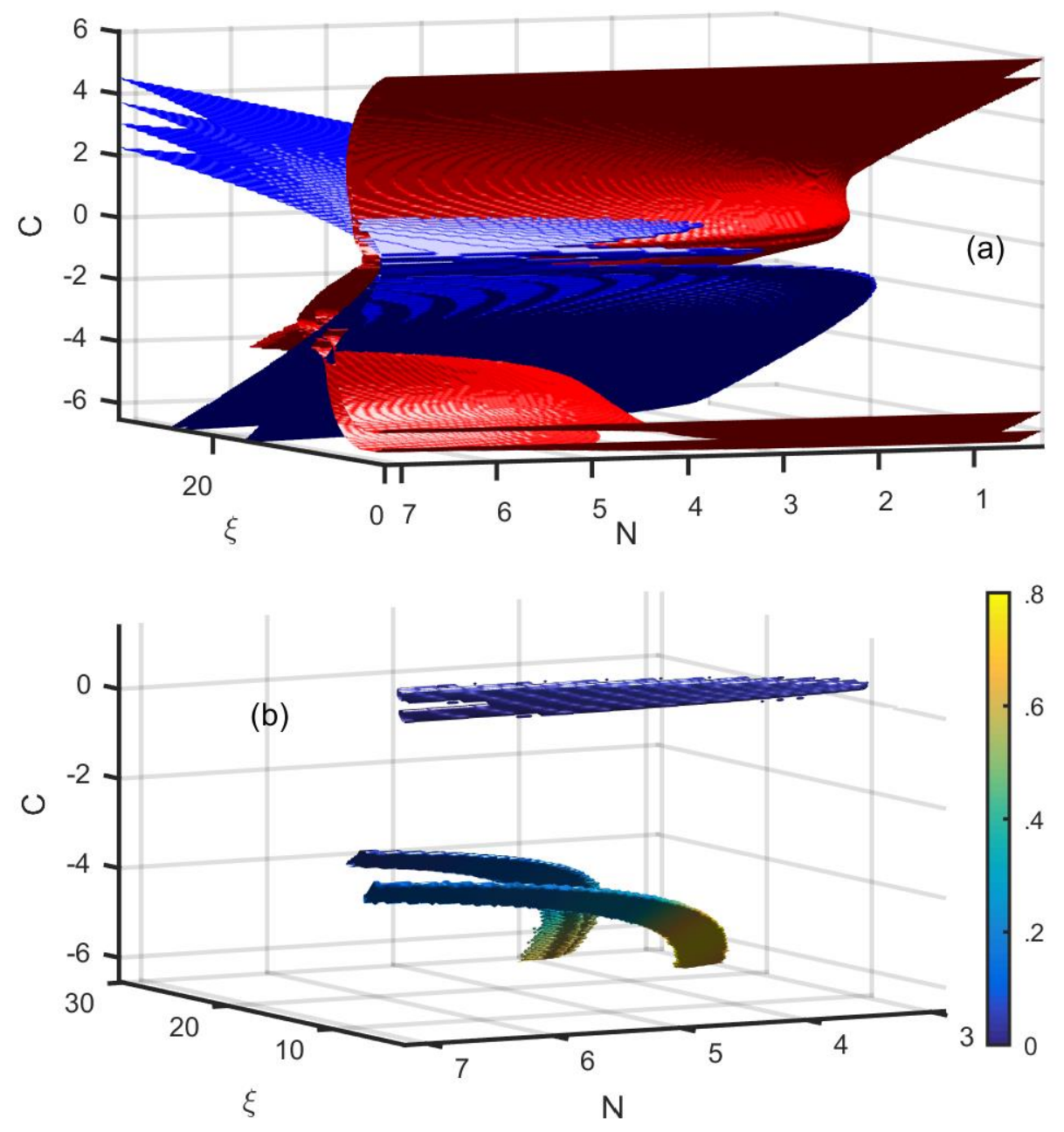

Figure 8: (a) Surfaces in the three-dimensional parameter space that enable the formation of a pulse shape with an excess kurtosis around -1 as typical of rectangular-like waveforms (blue), and an output temporal duration of $T_{\text {out }}=$ $20 T_{i n}$ (red). (b) Crossing of the two surfaces. The color-map indicates the Strehl ratio's values of the resulting pulses.

The temporal and spectral characteristics of the pulse generated at the point $(C=-6.1, N=3.2, \xi$ =23) are shown in Fig. 9 and highlight the effectiveness of the nonlinear reshaping process. The resulting waveform indeed features a very large and flat plateau and a fwhm width that is in agreement with the target value. The Strehl ratio of the pulse is 0.75 , and its spectral intensity profile is indeed close to that characteristic of a transform-limited pulse. These are signatures of the strong spectral compression process that characterizes the nonlinear pulse dynamics in the fiber in the operational regime being considered [27, 47]. 

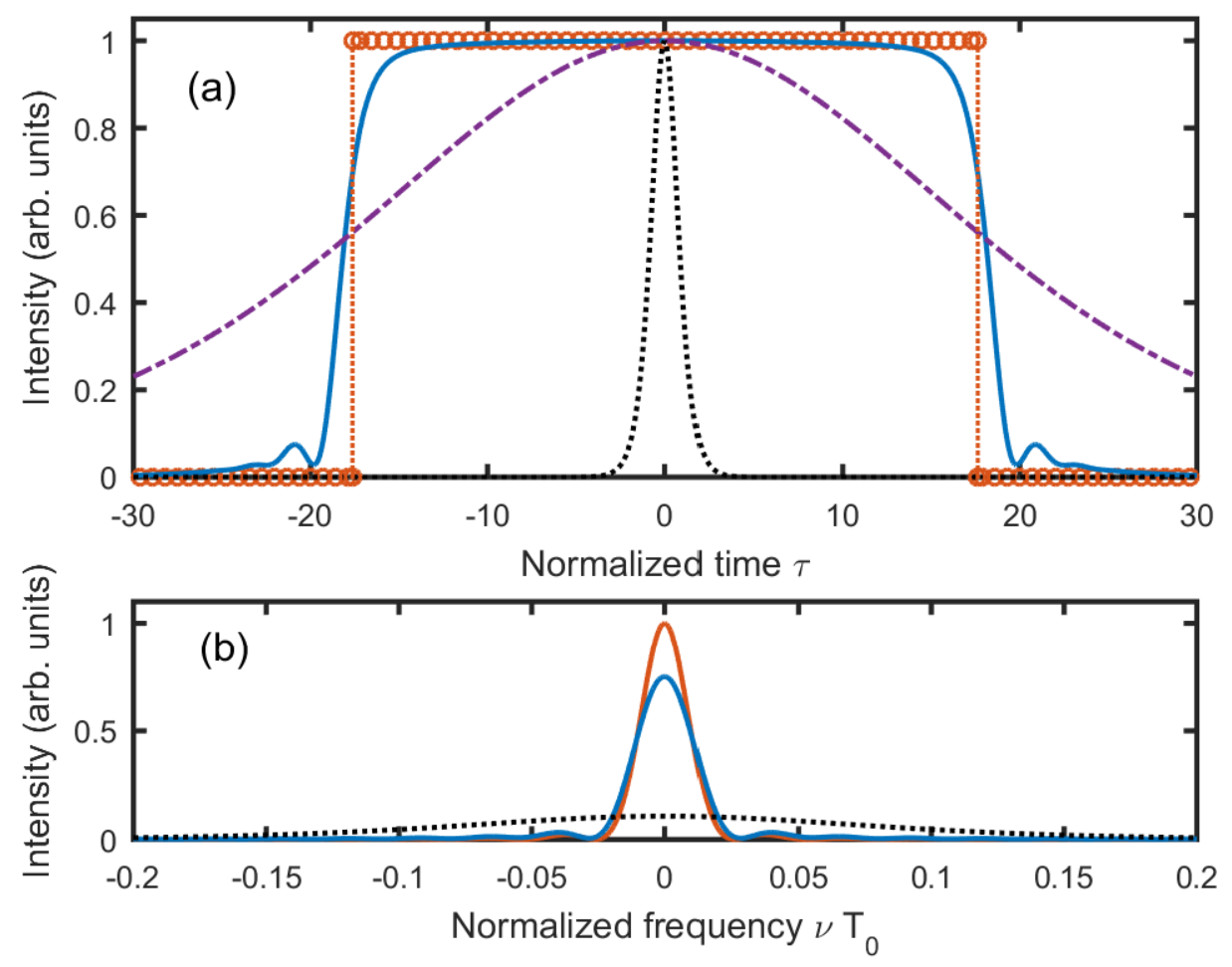

Figure 9: (a) Temporal and (b) spectral intensity profiles of the pulse generated at the point $(C=-6.5, N=3.2, \xi=$ 23) (blue curves). Also shown are the target temporal profile (red open circles), the pulse spectrum obtained assuming a flat temporal phase (solid red curve), the temporal and spectral profiles of the input pulse to the system (dotted black curves), and the temporal profile obtained after the initial dispersive propagation stage (dash-dotted purple curve).

\section{Conclusion}

We have presented a general approach to determine the parameters of fiber-based nonlinear pulse shaping systems that are required to generate pulses with preset temporal features. We have numerically shown that propagating an initial conventional laser pulse, such as a Gaussian or hyperbolic secant pulse, through a linear dispersive element followed by a nonlinear and normally dispersive fiber, it is possible to generate parabolic, triangular and nearly rectangular pulse waveforms of the desired duration and with limited chirp. Within our approach, the nonlinear shaping process is reduced to a numerical optimization problem over a three-dimensional space, where the intersections of different surfaces provide the means to quickly identify the sets of parameters of interest. We have also shown that this optimization problem can be solved efficiently by application of a regression model based on a neural network algorithm. Given the relatively low complexity of the system being studied in this work, the use of machine-learning approaches 
is not imperative, but one can easily imagine different fiber setups featuring additional adjustable parameters such as third-order dispersion or the presence of gain/loss.

In this paper, we have discussed the use of a silica fiber as the nonlinear shaping element, but the method described can be extended to waveguides fabricated in different nonlinear materials, such as silicon fibers [48, 49], and silicon or chalcogenide waveguides. Our proposed approach can also be extended to beam shaping [50]. In both these cases, additional effects such two-photon absorption and the presence of free carriers in the material should be included in the model, which would make extensive numerical simulations of the governing equations a costly exercise. In such cases, the use of a neural network training method could provide useful insights into a preliminary design of the system.

\section{Acknowledgements}

We acknowledge the support of the Institut Universitaire de France (IUF) and the Agence Nationale de la Recherche (Labex ACTION program ANR-11-LABX-01-01).

\section{References}

1. A. Chong, L. G. Wright, and F. W. Wise, "Ultrafast fiber lasers based on self-similar pulse evolution: a review of current progress," Rep. Prog. Phys. 78, 113901 (2015).

2. S. Boscolo and S. K. Turitsyn, "Intermediate asymptotics in nonlinear optical systems," Phys. Rev. A 85, 043811 (2012).

3. S. Boscolo, J. Peng, and C. Finot, "Design and Applications of In-Cavity Pulse Shaping by Spectral Sculpturing in Mode-Locked Fibre Lasers," Appl. Sci. 5, 1379 (2015).

4. L. Jing, N. Tigang, P. Li, J. Wei, Y. Haidong, C. Hongyao, and Z. Chan, "Photonic-Assisted Periodic Triangular-Shaped Pulses Generation With Tunable Repetition Rate," IEEE Photon. Technol. Lett. 25, 952-954 (2013).

5. F. Zhang, X. Ge, and S. Pan, "Triangular pulse generation using a dual-parallel Mach-Zehnder modulator driven by a single-frequency radio frequency signal," Opt. Lett. 38, 4491-4493 (2013).

6. W. Li, W. T. Wang, W. H. Sun, W. Y. Wang, and N. H. Zhu, "Generation of triangular waveforms based on a microwave photonic filter with negative coefficient," Opt. Express 22, 14993-15001 (2014).

7. J. Ye, L. Yan, W. Pan, B. Luo, X. Zou, A. Yi, and S. Yao, "Photonic generation of triangularshaped pulses bases on frequency to time conversion," Opt. Lett. 36, 1458-1460 (2011). 
8. J. Huh and J. Azaña, "Generation of high-quality parabolic pulses with optimized duration and energy by use of dispersive frequency-to-time mapping," Opt. Express 23, 27751-27762 (2015).

9. H.-S. Chan, Z.-M. Hsieh, W.-H. Liang, A. Kung, C.-K. Lee, C.-J. Lai, R.-P. Pan, and L.-H. Peng, "Synthesis and measurement of ultrafast waveforms from five discrete optical harmonics," Science 331, 1165-1168 (2011).

10. D. S. Wu, D. J. Richardson, and R. Slavík, "Optical Fourier synthesis of high-repetition-rate pulses," Optica 2, 18-26 (2015).

11. C. Finot, "40-GHz photonic waveform generator by linear shaping of four spectral sidebands," Opt. Lett. 40, 1422-1425 (2015).

12. S. T. Cundiff and A. M. Weiner, "Optical arbitrary waveform generation," Nat. Photonics 4, 760-766 (2010).

13. A. M. Clarke, D. G. Williams, M. A. F. Roelens, and B. J. Eggleton, "Reconfigurable Optical Pulse Generator Employing a Fourier-Domain Programmable Optical Processor," J. Lightw. Technol. 28, 97-103 (2010).

14. F. Parmigiani, P. Petropoulos, M. Ibsen, and D. J. Richardson, "Pulse retiming based on XPM using parabolic pulses formed in a fiber Bragg grating," IEEE Photon. Technol. Lett. 18, 829-831 (2006).

15. E. R. Andresen, J. M. Dudley, C. Finot, D. Oron, and H. Rigneault, "Transform-limited spectral compression by self-phase modulation of amplitude shaped pulses with negative chirp," Opt. Lett. 36, 707-709 (2011).

16. T. Hirooka, M. Nakazawa, and K. Okamoto, "Bright and dark $40 \mathrm{GHz}$ parabolic pulse generation using a picosecond optical pulse train and an arrayed waveguide grating," Opt. Lett. 33, 1102-1104 (2008).

17. S. Boscolo and C. Finot, Shaping Light in Nonlinear Optical Fibers (2017).

18. C. Finot, L. Provost, P. Petropoulos, and D. J. Richardson, "Parabolic pulse generation through passive nonlinear pulse reshaping in a normally dispersive two segment fiber device," Opt. Express 15, 852-864 (2007).

19. S. Pitois, C. Finot, J. Fatome, and G. Millot, "Generation of 20-Ghz picosecond pulse trains in the normal and anomalous dispersion regimes of optical fibers," Opt. Commun. 260, 301306 (2006).

20. A. Zeytunyan, G. Yesayan, L. Mouradian, P. Kockaert, P. Emplit, F. Louradour, and A. Barthélémy, "Nonlinear-dispersive similariton of passive fiber," J. Europ. Opt. Soc. Rap. Public. 4, 09009 (2009).

21. S. O. Iakushev, O. V. Shulika, and I. A. Sukhoivanov, "Passive nonlinear reshaping towards parabolic pulses in the steady-state regime in optical fibers," Opt. Commun. 285, 44934499 (2012).

22. S. Boscolo, A. I. Latkin, and S. K. Turitsyn, "Passive nonlinear pulse shaping in normally dispersive fiber systems," IEEE J. Quantum Electron. 44, 1196-1203 (2008).

23. H. Wang, A. I. Latkin, S. Boscolo, P. Harper, and S. K. Turitsyn, "Generation of triangularshaped optical pulses in normally dispersive fibre," J. Opt. 12, 035205 (2010).

24. N. Verscheure and C. Finot, "Pulse doubling and wavelength conversion through triangular nonlinear pulse reshaping," Electron. Lett. 47, 1194-1196 (2011).

25. B. G. Bale, S. Boscolo, K. Hammani, and C. Finot, "Effects of fourth-order fiber dispersion on ultrashort parabolic optical pulses in the normal dispersion regime," J. Opt. Soc. Am. B 28, 2059-2065 (2011). 
26. S. O. Iakushev, O. V. Shulika, I. A. Sukhoivanov, V. I. Fesenko, M. V. Andrés, and H. Sayinc, "Formation of ultrashort triangular pulses in optical fibers," Optics Express 22, 2911929134 (2014).

27. M. A. Kalashyan, K. A. Palandzhyan, G. L. Esayan, and L. K. Muradyan, "Generation of transform-limited rectangular pulses in a spectral compressor," Quantum Electron. 40, 868 (2010).

28. M. Oberthaler and R. A. Höpfel, "Spectral narrowing of ultrashort laser pulses by self-phase modulation in optical fibers," Appl. Phys. Lett. 63, 1017-1019 (1993).

29. C. Finot and S. Boscolo, "Design rules for nonlinear spectral compression in optical fibers," J. Opt. Soc. Am. B 33, 760-767 (2016).

30. D. Anderson, M. Desaix, M. Lisak, and M. L. Quiroga-Teixeiro, "Wave-breaking in nonlinear optical fibers," J. Opt. Soc. Am. B 9, 1358-1361 (1992).

31. T. Hirooka and M. Nakazawa, "Parabolic pulse generation by use of a dispersion-decreasing fiber with normal group-velocity dispersion," Opt. Lett. 29, 498-500 (2004).

32. N. G. R. Broderick, "Methods for pulse transformations using dispersion varying optical fibre tapers," Opt. Express 18, 24060-24069 (2010).

33. S. Wabnitz and C. Finot, "Theory of parabolic pulse propagation in nonlinear dispersion decreasing optical fiber amplifiers," J. Opt. Soc. Am. B 25, 614-621 (2008).

34. G. P. Agrawal, Nonlinear Fiber Optics, Fourth Edition (Academic Press, San Francisco, CA, 2006).

35. L. T. DeCarlo, "On the meaning and use of kurtosis," Psychol. Methods 2, 292-307 (1997).

36. B. Burgoyne, N. Godbout, and S. Lacroix, "Nonlinear pulse propagation in optical fibers using second order moments," Opt. Express 15, 10075-10090 (2007).

37. C.-K. Rosenberg, D. Anderson, M. Desaix, P. Johannisson, and M. Lisak, "Evolution of optical pulses towards wave breaking in highly nonlinear fibres," Opt. Commun. 273, 272-277 (2007).

38. M. Pawlowska, A. Patas, G. Achazi, and A. Lindinger, "Parametrically shaped femtosecond pulses in the nonlinear regime obtained by reverse propagation in an optical fiber," Opt. Lett. 37, 2709-2711 (2012).

39. X. Yang, D. J. Richardson, and P. Petropoulos, "Nonlinear Generation of Ultra-Flat Broadened Spectrum Based on Adaptive Pulse Shaping," J. Lightw. Technol. 30, 1971-1977 (2012).

40. A. Efimov, M. D. Moores, B. Mei, J. L. Krause, C. W. Siders, and D. H. Reitze, "Minimization of dispersion in an ultrafast chirped pulse amplifier using adaptive learning," Appl. Phys. B 70, S133-S141 (2000).

41. L. Michaeli and A. Bahabad, "Genetic algorithm driven spectral shaping of supercontinuum radiation in a photonic crystal fiber," J. Opt 20, 055501 (2018).

42. T. Baumeister, S. L. Brunton, and J. N. Kutz, "Deep learning and model predictive control for self-tuning mode-locked lasers," J. Opt. Soc. Am. B 35, 617-626 (2018).

43. M. E. Fermann, V. I. Kruglov, B. C. Thomsen, J. M. Dudley, and J. D. Harvey, "Self-similar propagation and amplification of parabolic pulses in optical fibers," Phys. Rev. Lett. 84, 6010-6013 (2000).

44. C. Finot, J. M. Dudley, B. Kibler, D. J. Richardson, and G. Millot, "Optical parabolic pulse generation and applications," IEEE J. Quantum Electron. 45, 1482-1489 (2009).

45. C. Finot, B. Kibler, L. Provost, and S. Wabnitz, "Beneficial impact of wave-breaking on coherent continuum formation in normally dispersive nonlinear fibers," J. Opt. Soc. Am. B 25, 1938-1948 (2008). 
46. A. Parriaux, M. Conforti, A. Bendahmane, J. Fatome, C. Finot, S. Trillo, N. Picqué, and G. Millot, "Spectral broadening of picosecond pulses forming dispersive shock waves in optical fibers," Opt. Lett. 42, 3044-3047 (2017).

47. A. A. Kutuzyan, T. G. Mansuryan, G. L. Esayan, R. S. Akobyan, and L. K. Mouradian, "Dispersive regime of spectral compression," Quantum Electron. 38, 383-387 (2008).

48. A. C. Peacock and N. Healy, "Parabolic pulse generation in tapered silicon fibers," Opt. Lett. 35, 1780-1782 (2010).

49. S. Lavdas, J. B. Driscoll, H. Jiang, R. R. Grote, R. M. Osgood, and N. C. Panoiu, "Generation of parabolic similaritons in tapered silicon photonic wires: comparison of pulse dynamics at telecom and mid-infrared wavelengths," Opt. Lett 38, 3953-3956 (2013).

50. A. R. Davoyan, S. K. Turitsyn, and Y. S. Kivshar, "Self-similar parabolic plasmonic beams," Opt. Lett. 38, 428-430 (2013). 